\title{
Trends in Analytical Instrument Making
}

DOI: $10.1134 / \mathrm{S} 1061934811020183$

An analytical instrument is a device with which an analytical method is implemented; however, the type of instrument, the originality of the technical solutions used, the reliability of its purely analytical characteristics, and the availability of instruments in themselves, to say nothing of their reasonable cost, substantially designate the potential of the method, its value, and interest in it.

New types of instruments appear regularly, which are either based on fundamentally new methods or revolutionize the implementation of existing ones. Thirty years ago nobody knew anything about ion mobility spectrometers; now they are service instruments in the army to detect poisonous and explosive substances. Inventions in mass spectrometers (orthogonal injection, mass reflectron, electrospray, inductively coupled plasma, etc.) have rapidly transformed mass spectrometry from a relatively exotic method, expensive and complex, into one of routine analysis.

Several trends, sometimes seemingly opposite, can be traced in the development of analytical instruments.

Therefore, on one hand, the sophistication of the analytical problems to be solved, the appearance of new problems, and the growth of requirements on sensitivity, selectivity, etc., give impetus to the development of complex and expensive, but powerful and smart analytical devices, e.g., mass spectrometry and its combination with chromatography and other separation methods. Instruments combining liquid chromatography with tandem mass spectrometry, Fourier transform ion cyclotron resonance mass spectrometers, and orbitrap mass spectrometers, widely known nowadays, possess remarkable analytical potential but are unavailable to many customers, not only for financial reasons.

On the other hand, there is a tendency to ensure routine analysis on simple-to-use and low-cost instruments, including for those for field and extralaboratory application. For this reason, numerous portable analyzers have been created, most often for one substance in a particular medium, for example, for meth- ane in coal mines, dissolved oxygen in water, and chlorine in the water of swimming pools.

The wide use of achievements in electronics and other engineering fields allows the creation of instruments that are not only more accurate, more multifunctional, etc., but also of smaller size. The miniaturization of analytical devices, computers among them, is one of the most noticeable trends. The advantages of small instruments are obvious: they take up less space, often becoming portable; and they consume less energy, reagents, and test materials. By now, the height of this direction is the development of microchipbased instruments.

A noticeable trend is the hyphenation of instruments, more exactly, their functions. One of the directions is a combination of two or several analytical methods in one device; examples are provided by complex and expensive instruments for studying surfaces, which combine electron probe microanalysis, $\mathrm{X}$-ray and Auger spectroscopy, and secondary-ion mass spectrometry in different combinations. One more version is the hyphenation of sample preparation and determination, as in flow-injection analysis and similar methods. Finally, there is the well-known combination of devices for the separation of mixtures and the subsequent determination of components, including almost all versions of analytical chromatography and capillary electrophoresis.

Speaking about the trends in analytical instrument making, we should not forget the block module principle of their design, when the customer himself can compose an instrument of separately purchased blocks. A chromatograph can be assembled from a pump, a detector, a column with an adsorbent, etc.

An engineer-instrument designer could add much more: about the wide use of lasers, including diode ones, especially in spectroscopy; detection arrays in spectroscopy; new data processing systems in instruments in themselves; spectral libraries incorporated into instruments; microprocessor control systems; and many others.

Yu. A. Zolotov 